\title{
A Hydrogen-bonded Organic Framework Based on
}

\section{Pyrazinopyrazine}

Qin Ji, ${ }^{\dagger}$ Kiyonori Takahashi, ${ }^{\dagger,}$ Shin-ichiro Noro, ${ }^{\mathbb{P}}$ Yusuke Ishigaki, ${ }^{\S}$ Kenta Kokado, ${ }^{\dagger+}$

Takayoshi Nakamura, ${ }^{\dagger}$ Ichiro Hisaki. ${ }^{\dagger \#}$

${ }^{\dagger}$ Graduate School of Environmental Science, Hokkaido University, Sapporo, Hokkaido 060-0810, Japan

${ }^{\sharp}$ Research Institute for Electronic Science, Hokkaido University, Sapporo, Hokkaido 001-0020, Japan.

"Faculty of Environmental Earth Science, Hokkaido University, Sapporo, Hokkaido 060-0810, Japan

${ }^{\S}$ Faculty of Science, Hokkaido University, Sapporo, Hokkaido 060-0810, Japan.

\# Graduate School of Engineering Science, Osaka University, 1-3 Machikaneyama, Toyonaka, Osaka 560-8531, Japan.

\section{Contents.}

1. Theoretical calculation.

2. Crystallography.

3. NMR spectroscopy. 


\section{Theoretical calculation}

Structural optimization and population analysis of CP-PP, ester derivative 5, and naphthalene analogue were performed by DFT at the B3LYP/6-31(d) level.

Table S1. Atomic coordinates of CP-PP

\begin{tabular}{|c|c|c|c|c|c|}
\hline \multirow{2}{*}{$\begin{array}{l}\text { Center } \\
\text { Number }\end{array}$} & \multirow{2}{*}{$\begin{array}{l}\text { Atomic } \\
\text { Number }\end{array}$} & \multirow{2}{*}{$\begin{array}{l}\text { Atomic } \\
\text { Type }\end{array}$} & \multicolumn{3}{|c|}{ Coordinates (Angstroms) } \\
\hline & & & $x$ & $\mathrm{Y}$ & Z \\
\hline 1 & 6 & 0 & 3.277593 & 0.161203 & 5.741615 \\
\hline 2 & 6 & 0 & 1.567032 & -0.002682 & 3.520940 \\
\hline 3 & 6 & 0 & 3.541130 & -0.758458 & 4.717135 \\
\hline 4 & 6 & 0 & 2.163928 & 1.005498 & 5.644715 \\
\hline 5 & 6 & 0 & 1.313161 & 0.920939 & 4.549134 \\
\hline 6 & 6 & 0 & 2.698867 & -0.829334 & 3.612824 \\
\hline 7 & 1 & 0 & 4.406204 & -1.408287 & 4.786334 \\
\hline 8 & 1 & 0 & 1.982430 & 1.723497 & 6.437396 \\
\hline 9 & 1 & 0 & 0.458444 & 1.585769 & 4.484026 \\
\hline 10 & 1 & 0 & 2.911420 & -1.520739 & 2.804473 \\
\hline 11 & 6 & 0 & 0.724776 & -0.063470 & 2.294452 \\
\hline 12 & 7 & 0 & -1.393145 & 0.192480 & 1.160184 \\
\hline 13 & 6 & 0 & 0.699899 & -0.118192 & 0.000000 \\
\hline 14 & 6 & 0 & -0.724776 & 0.063470 & 2.294452 \\
\hline 15 & 6 & 0 & -0.699899 & 0.118192 & 0.000000 \\
\hline 16 & 7 & 0 & 1.393145 & -0.192480 & -1.160184 \\
\hline 17 & 6 & 0 & 0.724776 & -0.063470 & -2.294452 \\
\hline 18 & 6 & 0 & -0.724776 & 0.063470 & -2.294452 \\
\hline 19 & 7 & 0 & -1.393145 & 0.192480 & -1.160184 \\
\hline 20 & 6 & 0 & -1.567032 & 0.002682 & 3.520940 \\
\hline 21 & 6 & 0 & -3.277593 & -0.161203 & 5.741615 \\
\hline 22 & 6 & 0 & -1.313161 & -0.920939 & 4.549134 \\
\hline 23 & 6 & 0 & -2.698867 & 0.829334 & 3.612824 \\
\hline 24 & 6 & 0 & -3.541130 & 0.758458 & 4.717135 \\
\hline 25 & 6 & 0 & -2.163928 & -1.005498 & 5.644715 \\
\hline 26 & 1 & 0 & -0.458444 & -1.585769 & 4.484026 \\
\hline 27 & 1 & 0 & -2.911420 & 1.520739 & 2.804473 \\
\hline 28 & 1 & 0 & -4.406204 & 1.408287 & 4.786334 \\
\hline 29 & 1 & 0 & -1.982430 & -1.723497 & 6.437396 \\
\hline 30 & 6 & 0 & -1.567032 & 0.002682 & -3.520940 \\
\hline 31 & 6 & 0 & -3.277593 & -0.161203 & -5.741615 \\
\hline 32 & 6 & 0 & -2.698867 & 0.829334 & -3.612824 \\
\hline 33 & 6 & 0 & -1.313161 & -0.920939 & -4.549134 \\
\hline 34 & 6 & 0 & -2.163928 & -1.005498 & -5.644715 \\
\hline 35 & 6 & 0 & -3.541130 & 0.758458 & -4.717135 \\
\hline 36 & 1 & 0 & -2.911420 & 1.520739 & -2.804473 \\
\hline 37 & 1 & 0 & -0.458444 & -1.585769 & -4.484026 \\
\hline 38 & 1 & 0 & -1.982430 & -1.723497 & -6.437396 \\
\hline 39 & 1 & 0 & -4.406204 & 1.408287 & -4.786334 \\
\hline 40 & 6 & 0 & 1.567032 & -0.002682 & -3.520940 \\
\hline 41 & 6 & 0 & 3.277593 & 0.161203 & -5.741615 \\
\hline 42 & 6 & 0 & 1.313161 & 0.920939 & -4.549134 \\
\hline 43 & 6 & 0 & 2.698867 & -0.829334 & -3.612824 \\
\hline 44 & 6 & 0 & 3.541130 & -0.758458 & -4.717135 \\
\hline 45 & 6 & 0 & 2.163928 & 1.005498 & -5.644715 \\
\hline 46 & 1 & 0 & 0.458444 & 1.585769 & -4.484026 \\
\hline 47 & 1 & 0 & 2.911420 & -1.520739 & -2.804473 \\
\hline 48 & 1 & 0 & 4.406204 & -1.408287 & -4.786334 \\
\hline 49 & 1 & 0 & 1.982430 & 1.723497 & -6.437396 \\
\hline 50 & 7 & 0 & 1.393145 & -0.192480 & 1.160184 \\
\hline 51 & 6 & 0 & -4.146951 & -0.288888 & 6.941640 \\
\hline 52 & 8 & 0 & -3.969674 & -1.079919 & 7.846151 \\
\hline
\end{tabular}




\begin{tabular}{|c|c|c|c|c|c|}
\hline 53 & 8 & 0 & -5.187615 & 0.582856 & 6.938258 \\
\hline 54 & 1 & 0 & -5.681003 & 0.407619 & 7.760942 \\
\hline 55 & 6 & 0 & 4.146951 & 0.288888 & 6.941640 \\
\hline 56 & 8 & 0 & 3.969674 & 1.079919 & 7.846151 \\
\hline 57 & 8 & 0 & 5.187615 & -0.582856 & 6.938258 \\
\hline 58 & 1 & 0 & 5.681003 & -0.407619 & 7.760942 \\
\hline 59 & 6 & 0 & -4.146951 & -0.288888 & -6.941640 \\
\hline 60 & 8 & 0 & -3.969674 & -1.079919 & -7.846151 \\
\hline 61 & 8 & 0 & -5.187615 & 0.582856 & -6.938258 \\
\hline 62 & 1 & 0 & -5.681003 & 0.407619 & -7.760942 \\
\hline 63 & 6 & 0 & 4.146951 & 0.288888 & -6.941640 \\
\hline 64 & 8 & 0 & 3.969674 & 1.079919 & -7.846151 \\
\hline 65 & 8 & 0 & 5.187615 & -0.582856 & -6.938258 \\
\hline 66 & 1 & 0 & 5.681003 & -0.407619 & -7.760942 \\
\hline
\end{tabular}


Table S2. Atomic coordinates of 5.

\begin{tabular}{|c|c|c|c|c|c|}
\hline \multirow{2}{*}{$\begin{array}{l}\text { Center } \\
\text { Number }\end{array}$} & \multirow{2}{*}{$\begin{array}{l}\text { Atomic } \\
\text { Number }\end{array}$} & \multirow{2}{*}{$\begin{array}{l}\text { Atomic } \\
\text { Type }\end{array}$} & \multicolumn{3}{|c|}{ Coordinates (Angstroms) } \\
\hline & & & $\mathrm{X}$ & $\mathrm{Y}$ & Z \\
\hline 1 & 6 & 0 & 3.275293 & 0.241332 & 5.742388 \\
\hline 2 & 6 & 0 & 1.566795 & 0.035919 & 3.521037 \\
\hline 3 & 6 & 0 & 3.561011 & -0.669116 & 4.715579 \\
\hline 4 & 6 & 0 & 2.139542 & 1.055698 & 5.646305 \\
\hline 5 & 6 & 0 & 1.290122 & 0.950607 & 4.551142 \\
\hline 6 & 6 & 0 & 2.720321 & -0.760398 & 3.611673 \\
\hline 7 & 1 & 0 & 4.442827 & -1.295883 & 4.783690 \\
\hline 8 & 1 & 0 & 1.939744 & 1.767569 & 6.440112 \\
\hline 9 & 1 & 0 & 0.418001 & 1.592707 & 4.488037 \\
\hline 10 & 1 & 0 & 2.950534 & -1.444731 & 2.802070 \\
\hline 11 & 6 & 0 & 0.726156 & -0.045543 & 2.294706 \\
\hline 12 & 7 & 0 & -1.397331 & 0.158616 & 1.160242 \\
\hline 13 & 6 & 0 & 0.702684 & -0.101511 & 0.000000 \\
\hline 14 & 6 & 0 & -0.726156 & 0.045543 & 2.294706 \\
\hline 15 & 6 & 0 & -0.702684 & 0.101511 & 0.000000 \\
\hline 16 & 7 & 0 & 1.397331 & -0.158616 & -1.160242 \\
\hline 17 & 6 & 0 & 0.726156 & -0.045543 & -2.294706 \\
\hline 18 & 6 & 0 & -0.726156 & 0.045543 & -2.294706 \\
\hline 19 & 7 & 0 & -1.397331 & 0.158616 & -1.160242 \\
\hline 20 & 6 & 0 & -1.566795 & -0.035919 & 3.521037 \\
\hline 21 & 6 & 0 & -3.275293 & -0.241332 & 5.742388 \\
\hline 22 & 6 & 0 & -1.290122 & -0.950607 & 4.551142 \\
\hline 23 & 6 & 0 & -2.720321 & 0.760398 & 3.611673 \\
\hline 24 & 6 & 0 & -3.561011 & 0.669116 & 4.715579 \\
\hline 25 & 6 & 0 & -2.139542 & -1.055698 & 5.646305 \\
\hline 26 & 1 & 0 & -0.418001 & -1.592707 & 4.488037 \\
\hline 27 & 1 & 0 & -2.950534 & 1.444731 & 2.802070 \\
\hline 28 & 1 & 0 & -4.442827 & 1.295883 & 4.783690 \\
\hline 29 & 1 & 0 & -1.939744 & -1.767569 & 6.440112 \\
\hline 30 & 6 & 0 & -1.566795 & -0.035919 & -3.521037 \\
\hline 31 & 6 & 0 & -3.275293 & -0.241332 & -5.742388 \\
\hline 32 & 6 & 0 & -2.720321 & 0.760398 & -3.611673 \\
\hline 33 & 6 & 0 & -1.290122 & -0.950607 & -4.551142 \\
\hline 34 & 6 & 0 & -2.139542 & -1.055698 & -5.646305 \\
\hline 35 & 6 & 0 & -3.561011 & 0.669116 & -4.715579 \\
\hline 36 & 1 & 0 & -2.950534 & 1.444731 & -2.802070 \\
\hline 37 & 1 & 0 & -0.418001 & -1.592707 & -4.488037 \\
\hline 38 & 1 & 0 & -1.939744 & -1.767569 & -6.440112 \\
\hline 39 & 1 & 0 & -4.442827 & 1.295883 & -4.783690 \\
\hline 40 & 6 & 0 & 1.566795 & 0.035919 & -3.521037 \\
\hline 41 & 6 & 0 & 3.275293 & 0.241332 & -5.742388 \\
\hline 42 & 6 & 0 & 1.290122 & 0.950607 & -4.551142 \\
\hline 43 & 6 & 0 & 2.720321 & -0.760398 & -3.611673 \\
\hline 44 & 6 & 0 & 3.561011 & -0.669116 & -4.715579 \\
\hline 45 & 6 & 0 & 2.139542 & 1.055698 & -5.646305 \\
\hline 46 & 1 & 0 & 0.418001 & 1.592707 & -4.488037 \\
\hline 47 & 1 & 0 & 2.950534 & -1.444731 & -2.802070 \\
\hline 48 & 1 & 0 & 4.442827 & -1.295883 & -4.783690 \\
\hline 49 & 1 & 0 & 1.939744 & 1.767569 & -6.440112 \\
\hline 50 & 7 & 0 & 1.397331 & -0.158616 & 1.160242 \\
\hline 51 & 6 & 0 & -4.141890 & -0.392864 & 6.946355 \\
\hline 52 & 8 & 0 & -3.935505 & -1.183961 & 7.845635 \\
\hline 53 & 8 & 0 & -5.196019 & 0.454216 & 6.933235 \\
\hline 54 & 6 & 0 & 4.141890 & 0.392864 & 6.946355 \\
\hline 55 & 8 & 0 & 3.935505 & 1.183961 & 7.845635 \\
\hline 56 & 8 & 0 & 5.196019 & -0.454216 & 6.933235 \\
\hline 57 & 6 & 0 & -4.141890 & -0.392864 & -6.946355 \\
\hline 58 & 8 & 0 & -3.935505 & -1.183961 & -7.845635 \\
\hline 59 & 8 & 0 & -5.196019 & 0.454216 & -6.933235 \\
\hline 60 & 6 & 0 & 4.141890 & 0.392864 & -6.946355 \\
\hline 61 & 8 & 0 & 3.935505 & 1.183961 & -7.845635 \\
\hline 62 & 8 & 0 & 5.196019 & -0.454216 & -6.933235 \\
\hline
\end{tabular}




\begin{tabular}{rrrrrr}
63 & 6 & 0 & 6.072908 & -0.357691 & 8.066400 \\
64 & 1 & 0 & 6.502802 & 0.645148 & 8.134422 \\
65 & 1 & 0 & 6.853373 & -1.099662 & 7.895365 \\
66 & 1 & 0 & 5.530592 & -0.574529 & 8.990492 \\
67 & 6 & 0 & -6.072908 & 0.357691 & 8.066400 \\
68 & 1 & 0 & -6.502802 & -0.645148 & 8.134422 \\
69 & 1 & 0 & -6.853373 & 1.099662 & 7.895365 \\
70 & 1 & 0 & -5.530592 & 0.574529 & 8.990492 \\
71 & 6 & 0 & -6.072908 & 0.357691 & -8.066400 \\
72 & 1 & 0 & -5.530592 & 0.574529 & -8.990492 \\
73 & 1 & 0 & -6.853373 & 1.099662 & -7.895365 \\
74 & 1 & 0 & -6.502802 & -0.645148 & -8.134422 \\
75 & 6 & 0 & 6.072908 & -0.357691 & -8.066400 \\
76 & 1 & 0 & 5.530592 & -0.574529 & -8.990492 \\
77 & 1 & 0 & 6.853373 & -1.099662 & -7.895365 \\
78 & 1 & 0 & 6.502802 & 0.645148 & -8.134422 \\
\hline
\end{tabular}


Table S3. Atomic coordinates of the naphthalene analogue.

\begin{tabular}{|c|c|c|c|c|c|}
\hline \multirow{2}{*}{$\begin{array}{l}\text { Center } \\
\text { Number }\end{array}$} & \multirow{2}{*}{$\begin{array}{l}\text { Atomic } \\
\text { Number }\end{array}$} & \multirow{2}{*}{$\begin{array}{l}\text { Atomic } \\
\text { Type }\end{array}$} & \multicolumn{3}{|c|}{ Coordinates (Angstroms) } \\
\hline & & & $x$ & Y & Z \\
\hline 1 & 6 & 0 & 3.170394 & 0.163102 & 5.991895 \\
\hline 2 & 6 & 0 & 1.532866 & 0.022921 & 3.703454 \\
\hline 3 & 6 & 0 & 3.382184 & -0.837440 & 5.034826 \\
\hline 4 & 6 & 0 & 2.138207 & 1.091934 & 5.800141 \\
\hline 5 & 6 & 0 & 1.328961 & 1.020696 & 4.673376 \\
\hline 6 & 6 & 0 & 2.572306 & -0.901396 & 3.904334 \\
\hline 7 & 1 & 0 & 4.175658 & -1.562157 & 5.179905 \\
\hline 8 & 1 & 0 & 1.989160 & 1.866137 & 6.545555 \\
\hline 9 & 1 & 0 & 0.538451 & 1.750913 & 4.533076 \\
\hline 10 & 1 & 0 & 2.734369 & -1.686593 & 3.171187 \\
\hline 11 & 6 & 0 & 0.719933 & -0.024267 & 2.455363 \\
\hline 12 & 6 & 0 & 0.711474 & -0.039856 & 0.000000 \\
\hline 13 & 6 & 0 & -0.719933 & 0.024267 & 2.455363 \\
\hline 14 & 6 & 0 & -0.711474 & 0.039856 & 0.000000 \\
\hline 15 & 6 & 0 & 0.719933 & -0.024267 & -2.455363 \\
\hline 16 & 6 & 0 & -0.719933 & 0.024267 & -2.455363 \\
\hline 17 & 6 & 0 & -1.532866 & -0.022921 & 3.703454 \\
\hline 18 & 6 & 0 & -3.170394 & -0.163102 & 5.991895 \\
\hline 19 & 6 & 0 & -1.328961 & -1.020696 & 4.673376 \\
\hline 20 & 6 & 0 & -2.572306 & 0.901396 & 3.904334 \\
\hline 21 & 6 & 0 & -3.382184 & 0.837440 & 5.034826 \\
\hline 22 & 6 & 0 & -2.138207 & -1.091934 & 5.800141 \\
\hline 23 & 1 & 0 & -0.538451 & -1.750913 & 4.533076 \\
\hline 24 & 1 & 0 & -2.734369 & 1.686593 & 3.171187 \\
\hline 25 & 1 & 0 & -4.175658 & 1.562157 & 5.179905 \\
\hline 26 & 1 & 0 & -1.989160 & -1.866137 & 6.545555 \\
\hline 27 & 6 & 0 & -1.532866 & -0.022921 & -3.703454 \\
\hline 28 & 6 & 0 & -3.170394 & -0.163102 & -5.991895 \\
\hline 29 & 6 & 0 & -2.572306 & 0.901396 & -3.904334 \\
\hline 30 & 6 & 0 & -1.328961 & -1.020696 & -4.673376 \\
\hline 31 & 6 & 0 & -2.138207 & -1.091934 & -5.800141 \\
\hline 32 & 6 & 0 & -3.382184 & 0.837440 & -5.034826 \\
\hline 33 & 1 & 0 & -2.734369 & 1.686593 & -3.171187 \\
\hline 34 & 1 & 0 & -0.538451 & -1.750913 & -4.533076 \\
\hline 35 & 1 & 0 & -1.989160 & -1.866137 & -6.545555 \\
\hline 36 & 1 & 0 & -4.175658 & 1.562157 & -5.179905 \\
\hline 37 & 6 & 0 & 1.532866 & 0.022921 & -3.703454 \\
\hline 38 & 6 & 0 & 3.170394 & 0.163102 & -5.991895 \\
\hline 39 & 6 & 0 & 1.328961 & 1.020696 & -4.673376 \\
\hline
\end{tabular}




\begin{tabular}{|c|c|c|c|c|c|}
\hline 40 & 6 & 0 & 2.572306 & -0.901396 & -3.904334 \\
\hline 41 & 6 & 0 & 3.382184 & -0.837440 & -5.034826 \\
\hline 42 & 6 & 0 & 2.138207 & 1.091934 & -5.800141 \\
\hline 43 & 1 & 0 & 0.538451 & 1.750913 & -4.533076 \\
\hline 44 & 1 & 0 & 2.734369 & -1.686593 & -3.171187 \\
\hline 45 & 1 & 0 & 4.175658 & -1.562157 & -5.179905 \\
\hline 46 & 1 & 0 & 1.989160 & 1.866137 & -6.545555 \\
\hline 47 & 6 & 0 & -4.003515 & -0.285662 & 7.215377 \\
\hline 48 & 8 & 0 & -3.866134 & -1.139178 & 8.069109 \\
\hline 49 & 8 & 0 & -4.966220 & 0.669652 & 7.301578 \\
\hline 50 & 6 & 0 & 4.003515 & 0.285662 & 7.215377 \\
\hline 51 & 8 & 0 & 3.866134 & 1.139178 & 8.069109 \\
\hline 52 & 8 & 0 & 4.966220 & -0.669652 & 7.301578 \\
\hline 53 & 6 & 0 & -4.003515 & -0.285662 & -7.215377 \\
\hline 54 & 8 & 0 & -3.866134 & -1.139178 & -8.069109 \\
\hline 55 & 8 & 0 & -4.966220 & 0.669652 & -7.301578 \\
\hline 56 & 6 & 0 & 4.003515 & 0.285662 & -7.215377 \\
\hline 57 & 8 & 0 & 3.866134 & 1.139178 & -8.069109 \\
\hline 58 & 8 & 0 & 4.966220 & -0.669652 & -7.301578 \\
\hline 59 & 6 & 0 & 1.389175 & -0.063815 & -1.243635 \\
\hline 60 & 6 & 0 & -1.389175 & 0.063815 & -1.243635 \\
\hline 61 & 6 & 0 & 1.389175 & -0.063815 & 1.243635 \\
\hline 62 & 6 & 0 & -1.389175 & 0.063815 & 1.243635 \\
\hline 63 & 1 & 0 & 5.440453 & -0.488613 & 8.134024 \\
\hline 64 & 1 & 0 & -5.440453 & 0.488613 & 8.134024 \\
\hline 65 & 1 & 0 & 5.440453 & -0.488613 & -8.134024 \\
\hline 66 & 1 & 0 & -5.440453 & 0.488613 & -8.134024 \\
\hline 67 & 1 & 0 & 2.476129 & -0.074744 & 1.241152 \\
\hline 68 & 1 & 0 & 2.476129 & -0.074744 & -1.241152 \\
\hline 69 & 1 & 0 & -2.476129 & 0.074744 & -1.241152 \\
\hline 70 & 1 & 0 & -2.476129 & 0.074744 & 1.241152 \\
\hline
\end{tabular}



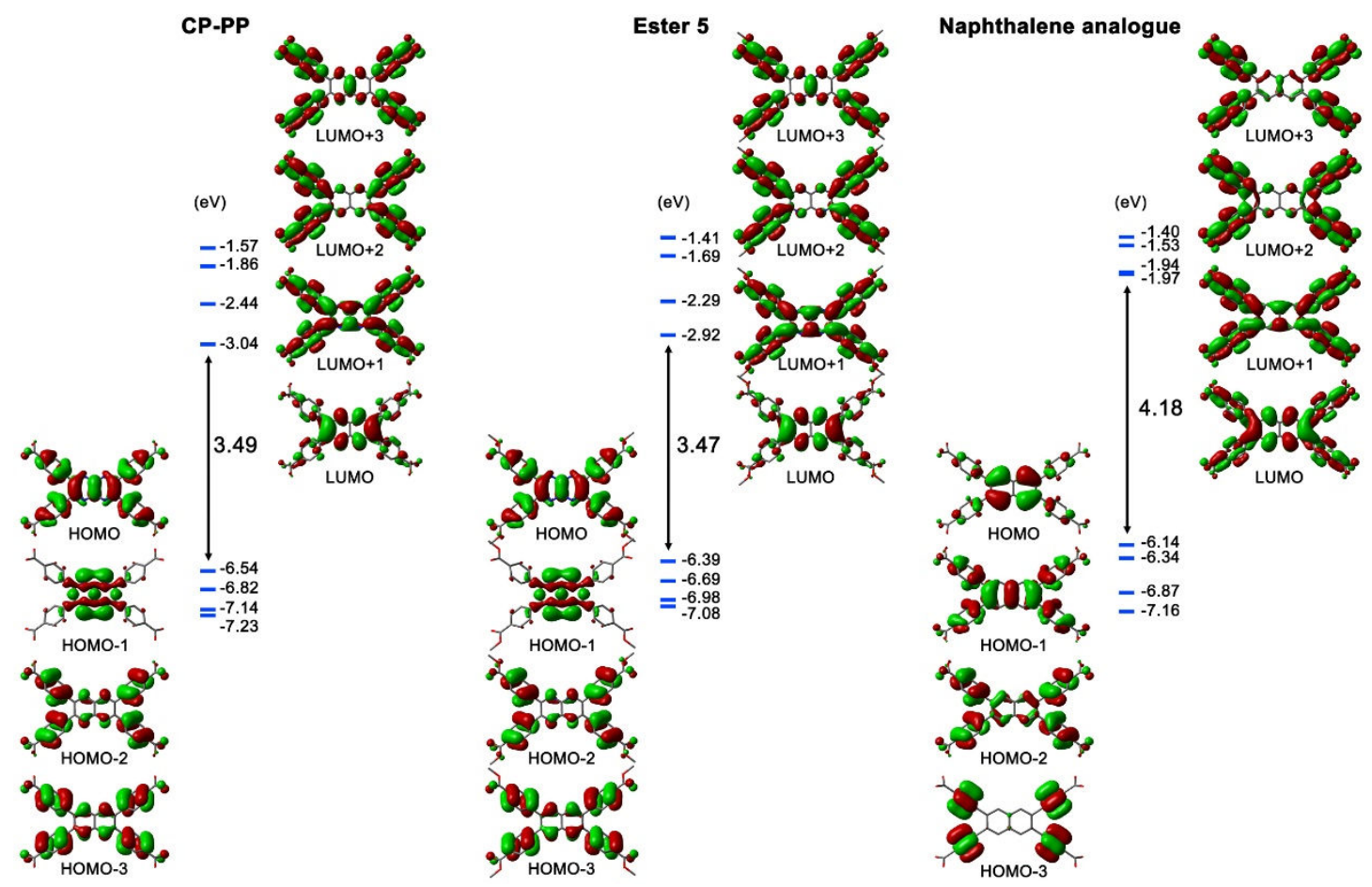

Figure S1. Selected molecular orbitals and energy levels of CP-PP, ester 5 and the naphthalene analogue calculated at the B3LYP/6-31G(d) level of theory. 


\section{Crystallography}

(a)

(b)

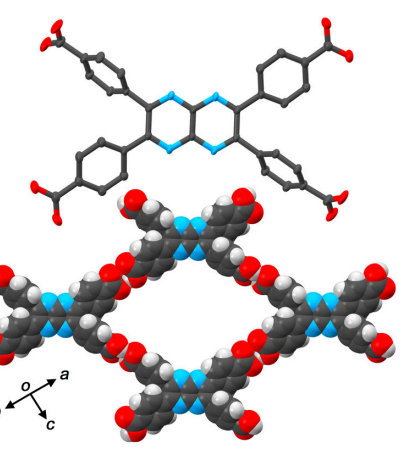

(c)

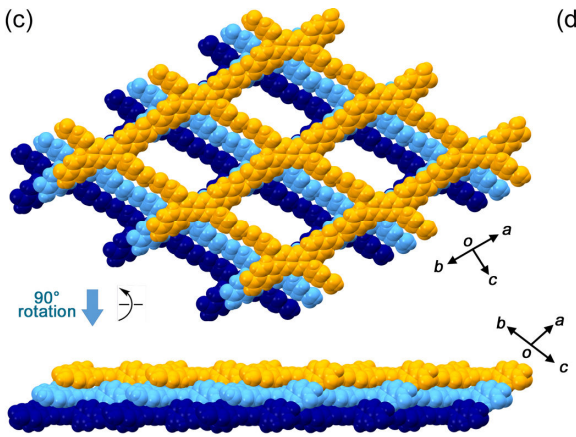

(d)

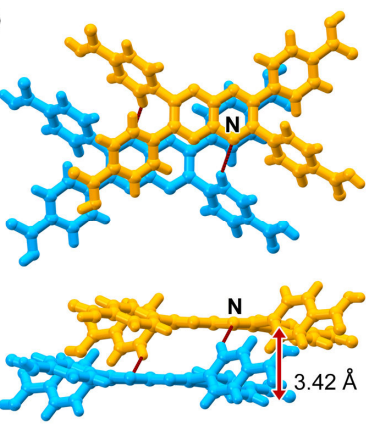

Figure S2. Crystal structure of CP-PP-1(TCB): (a) Molecular structure drawn with anisotropic displacement ellipsoids with 50\% provability. (b) Hydrogen-bonded network motif composed of four molecules. (c) Selected layered structure stacked with an inclined AA pattern. (d) Orientation of two adjacent molecules. The crystal structure is closely similar with that of CP-PP-1(DCB), while subtle differences such as symmetry of the molecule of CP-PP, the dihedral angle between the PP core and peripheral phenylene rings, $\mathrm{N} \cdots \mathrm{C}-\mathrm{H}$ interactions are observed.

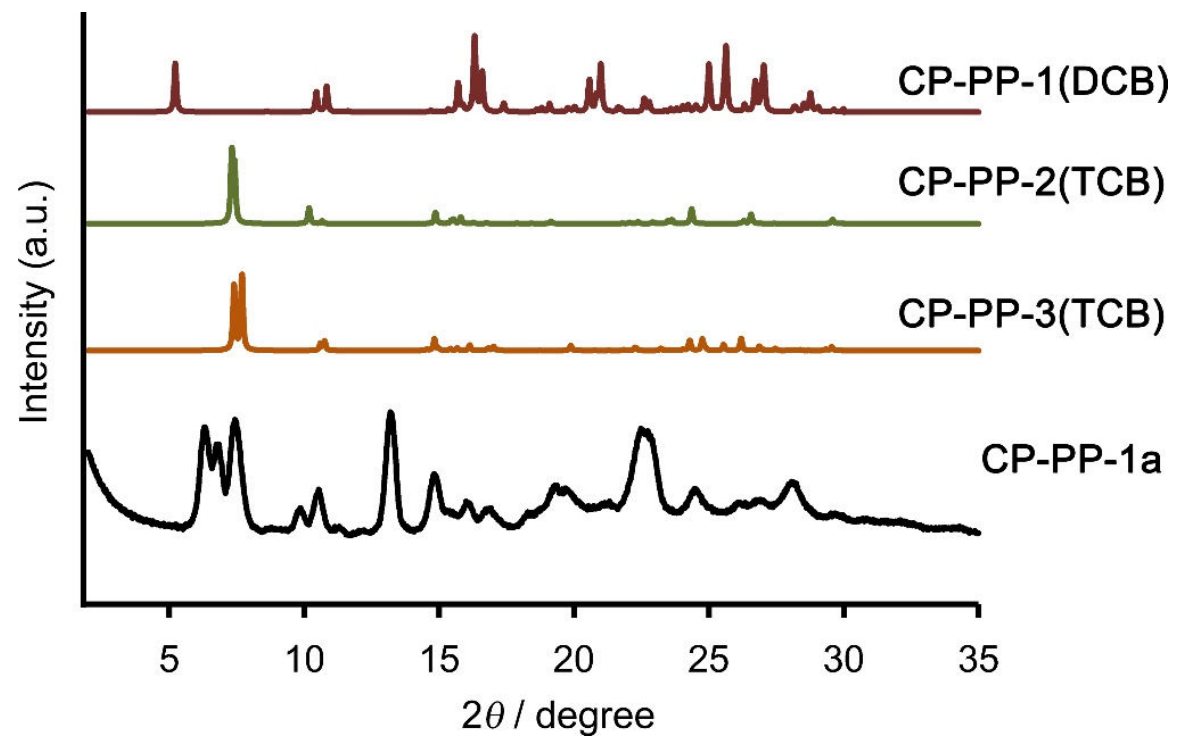

Figure S3. A PXRD pattern of activated HOF CP-PP-1a (bottom), together with simulated patterns of CP-PP-1(DCB), CP-PP-2(TCB), and CP-PP-3(TCB) (top). 
(a)

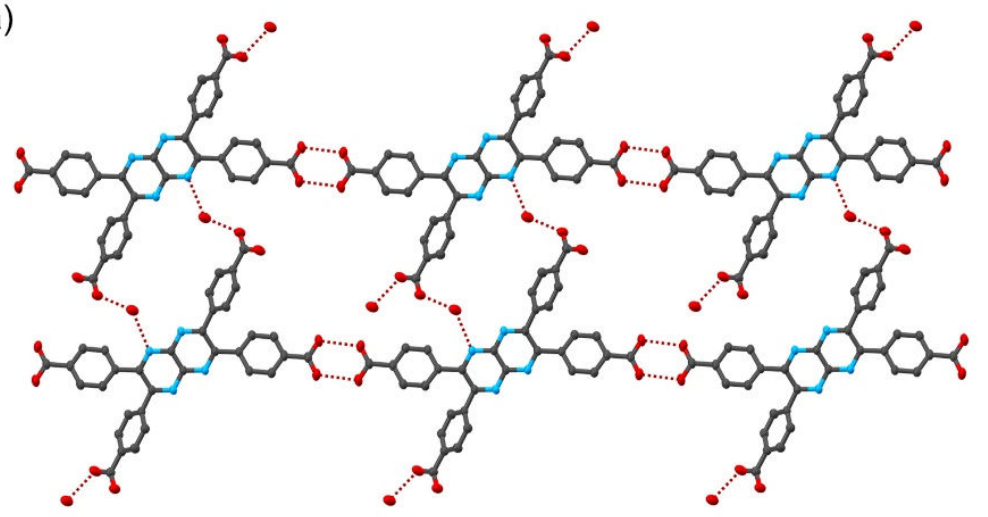

(b)

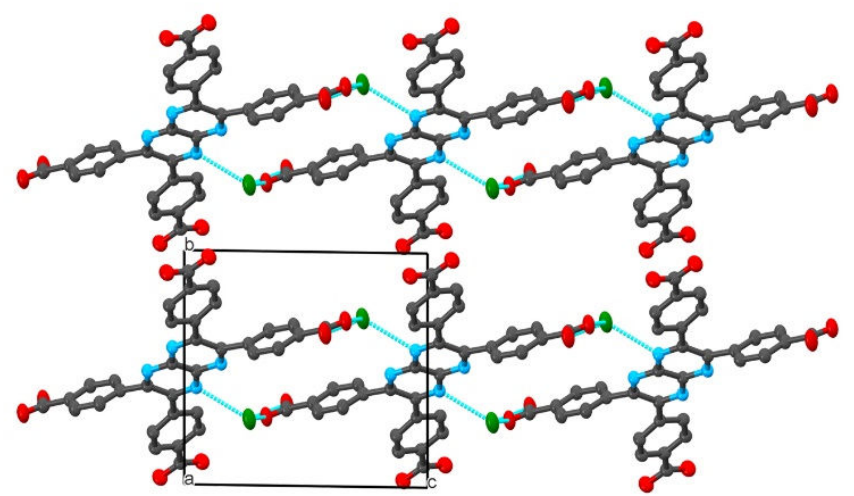

Figure S4. Crystal structure of CP-PP-3(TCB). (a) A layer structure composed of CP-PP and water molecules connected through H-bonds. The water molecules also form H-bonds with CP-PP molecules of the neighboring layers. (b) Packing diagram viewed down from the $a$ axis. The water molecules are colored green for clarity. 


\section{NMR spectroscopy.}

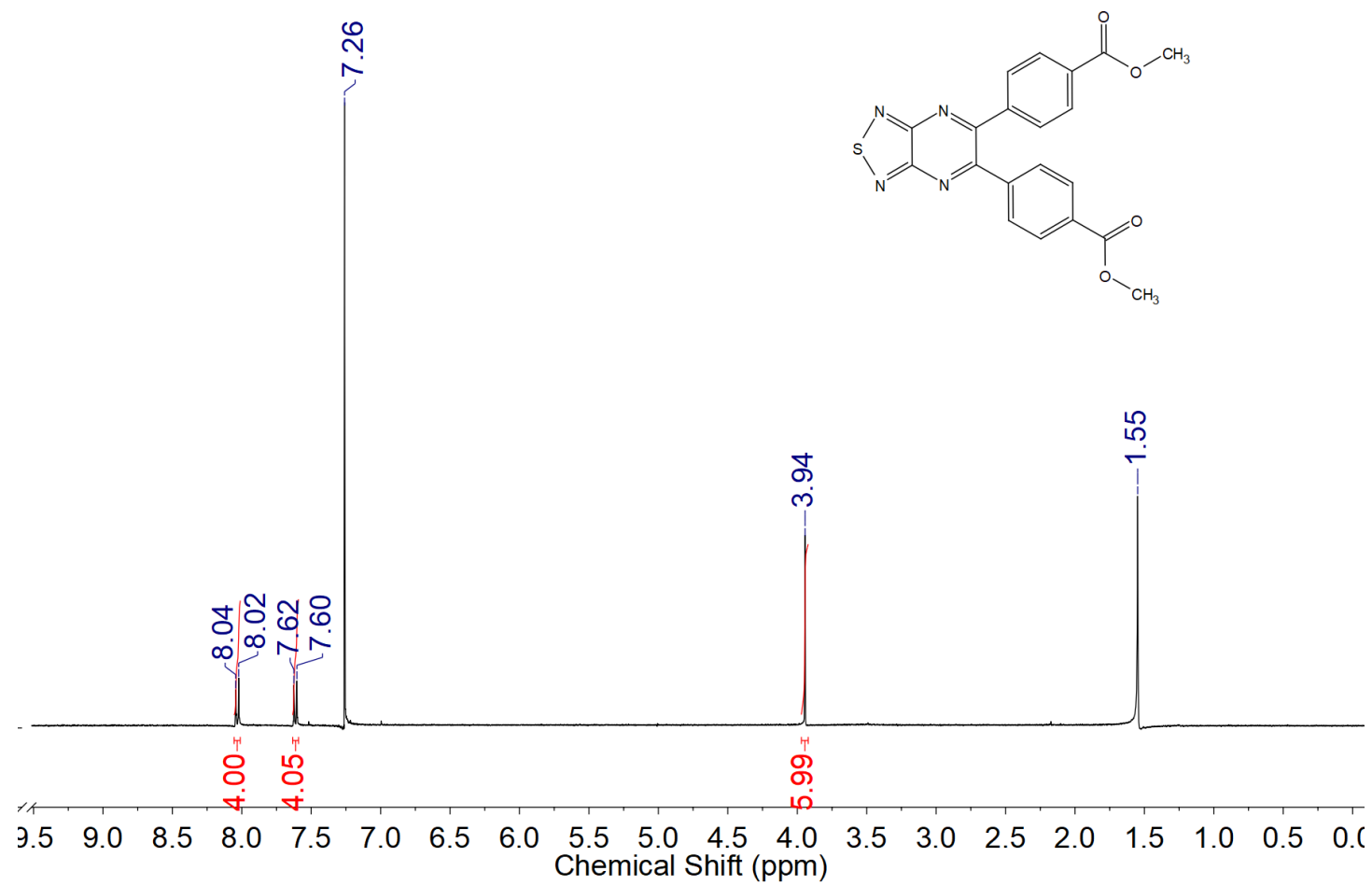

Figure S5. ${ }^{1} \mathrm{H} \mathrm{NMR}\left(400 \mathrm{MHz}, \mathrm{CDCl}_{3}\right)$ spectrum of 3.

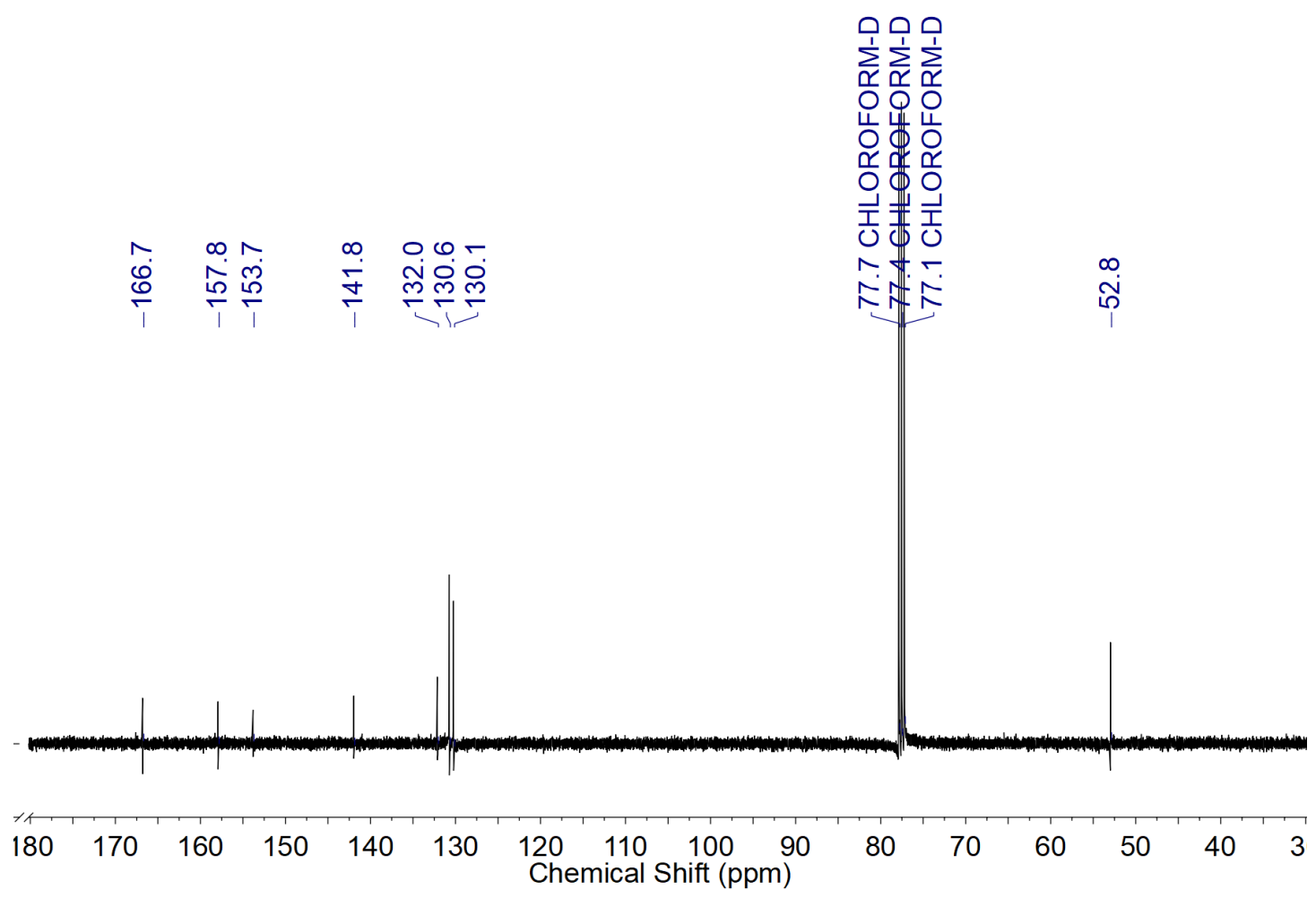

Figure S6. ${ }^{13} \mathrm{C}$ NMR $\left(100 \mathrm{MHz}, \mathrm{CDCl}_{3}\right)$ spectrum of 3 . 


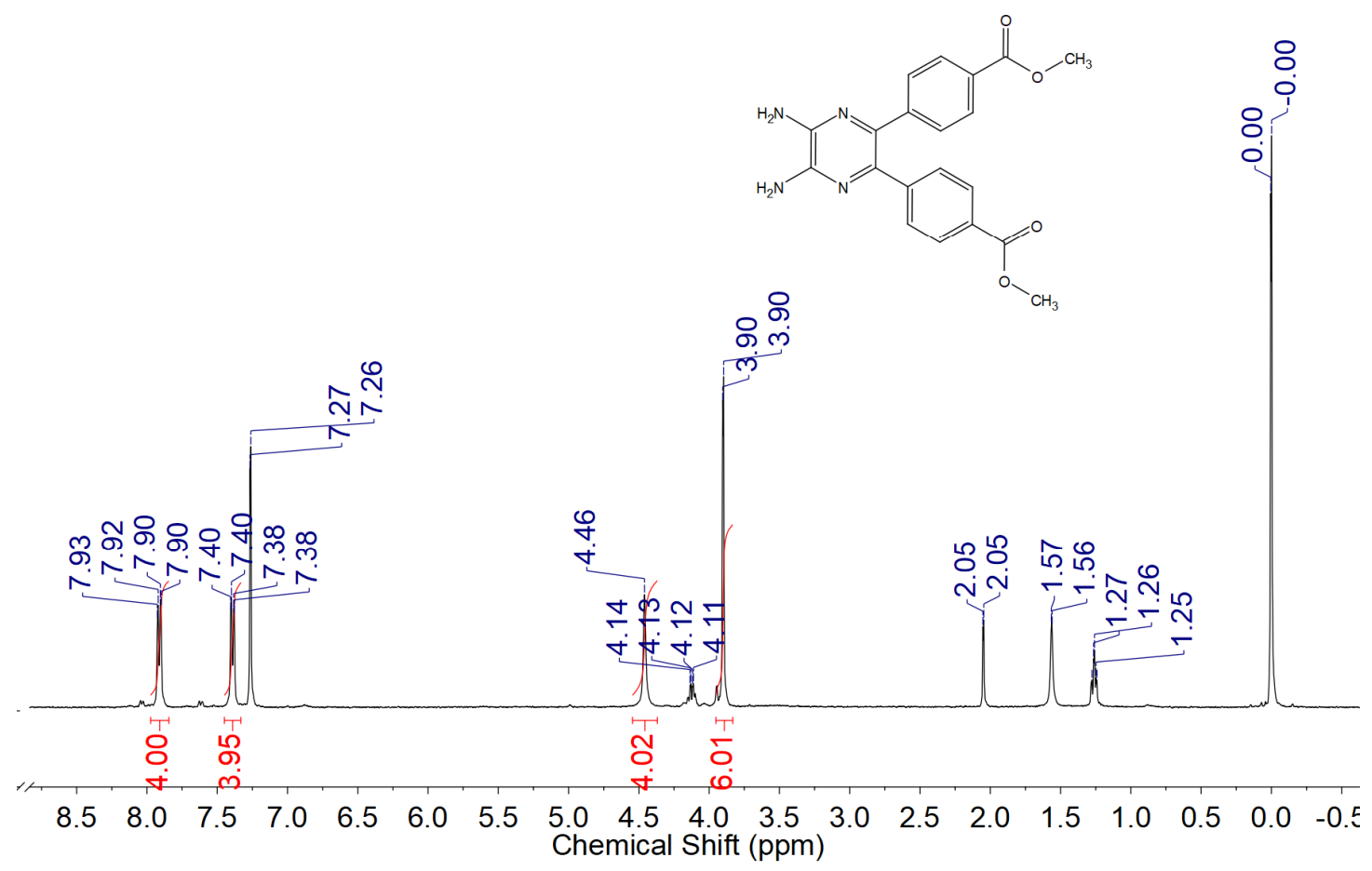

Figure S7. ${ }^{1} \mathrm{H}$ NMR $\left(400 \mathrm{MHz}, \mathrm{CDCl}_{3}\right)$ spectrum of 4 .
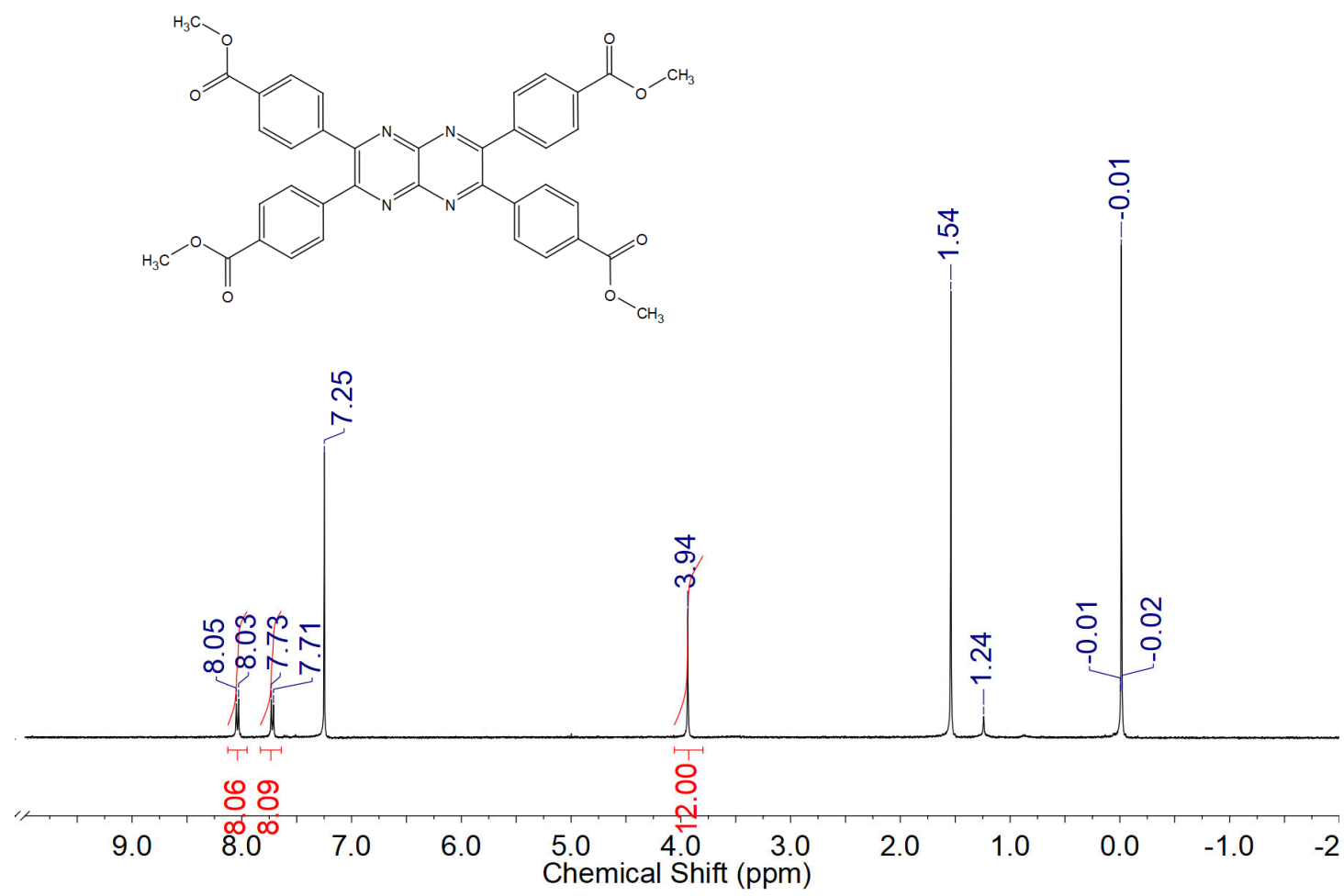

Figure S8. ${ }^{1} \mathrm{H}$ NMR $\left(400 \mathrm{MHz}, \mathrm{CDCl}_{3}\right)$ spectrum of 5 . 
N $\infty \quad N+\sim 6$

๑

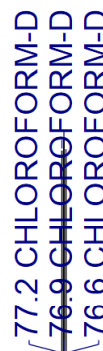

NN

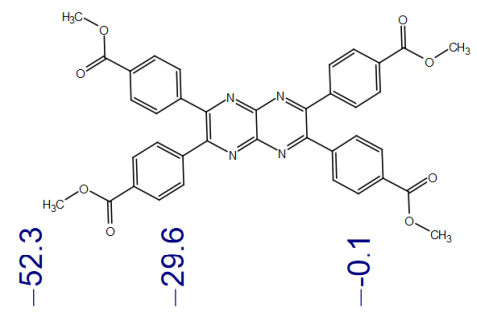

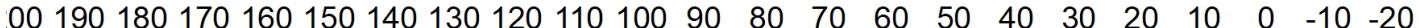
Chemical Shift (ppm)

Figure S9. ${ }^{13} \mathrm{C} \mathrm{NMR}\left(100 \mathrm{MHz}, \mathrm{CDCl}_{3}\right)$ spectrum of 5.

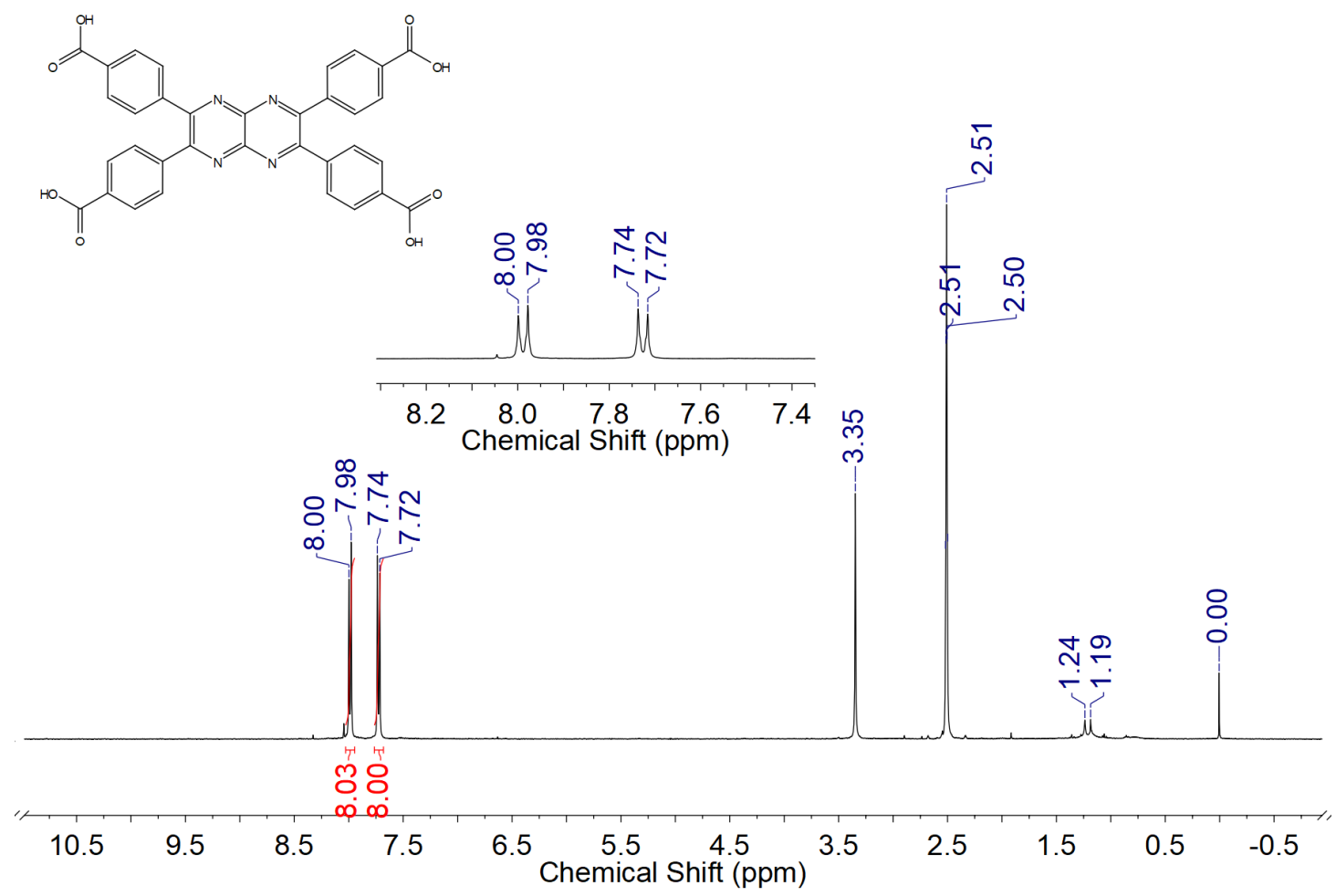

Figure S10. ${ }^{1} \mathrm{H}$ NMR (400 MHz, DMSO- $d_{6}$ ) spectrum of CP-PP. 


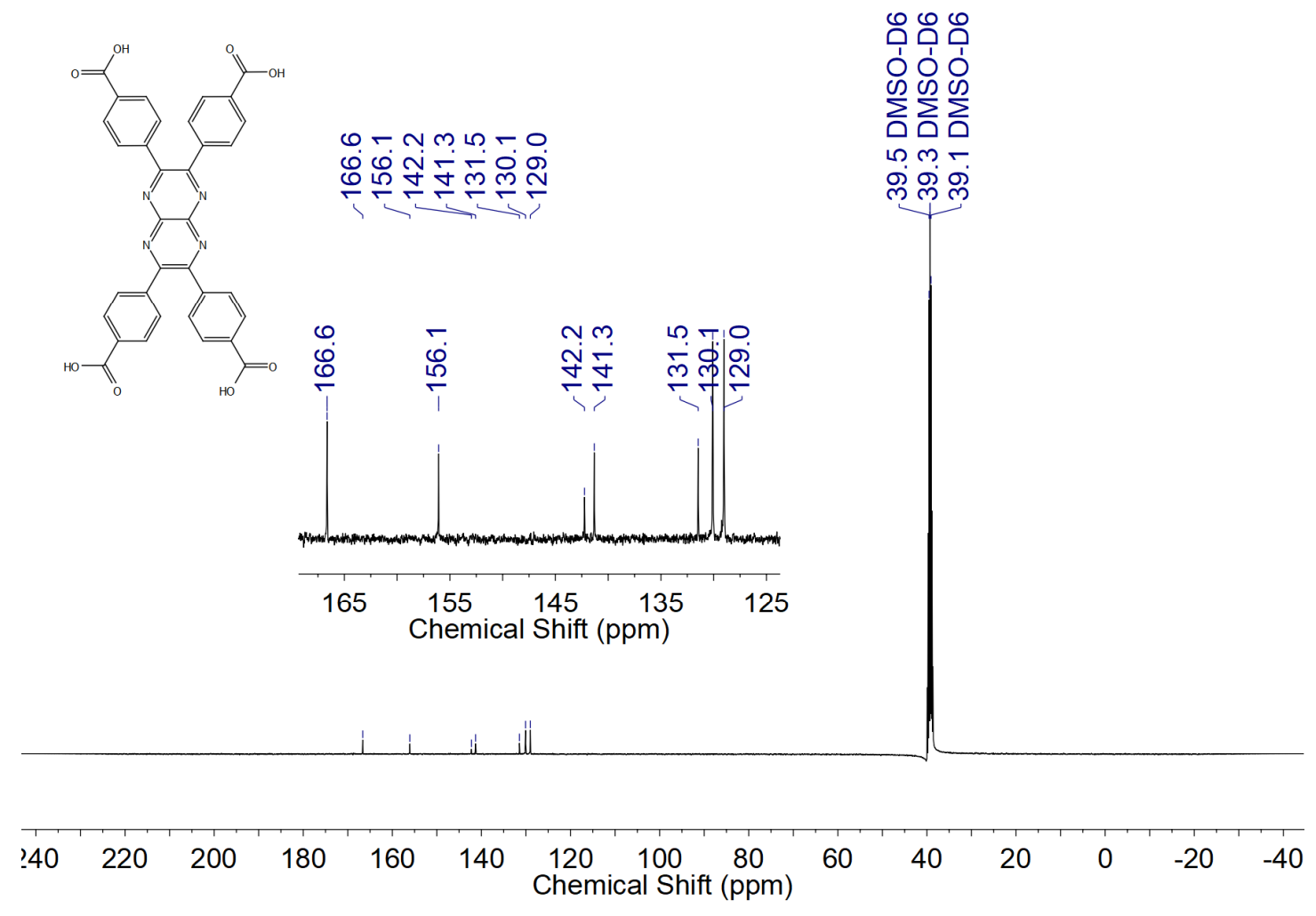

Figure S11. ${ }^{13} \mathrm{C}$ NMR (100 MHz, DMSO- $\left.d_{6}\right)$ spectrum of CP-PP. 